\title{
Enfermedades inflamatorias intestinales: Experiencia de dos centros chilenos
}

\author{
Carolina Figueroa $C^{1}$, Rodrigo $Q$ uera $\mathbf{P}^{1-3}$, Jorge \\ Valenzuela $E^{2,3}$, Christian Jensen $B^{4}$. \\ Inflammatory bowel disease: \\ Experience of two Chilean centers
}

Background: The incidence of Inflammatory Bowel Disease (IBD) has increased, particularly in the developed world, and probably also in Chile. Aim: To report our experience with a large group of patients from two medical centers, Hospital Clinico de la Universidad de Chile (HCUCH) and Clinica Las Condes (CLC) and to characterize the clinical features of IBD in Chile. Patients and methods: Retrospective review of 238 patients with IBD (age range 14-78 years, 143 women) treated at HCUCH and CLC between January 1990 and August 2002. Results: One hundred eighty one patients had ulcerative colitis (UC) and 57 had Crohn's disease (CD), with an UC/CD ratio of 3.2/1. Forty eight percent of patients were aged between 20 and 39 years old and $69 \%$ were diagnosed after 1995. Patients from HCUCH had more severe disease, more complications and needed surgery more often. Conclusions: Clinical features of Chilean patients with IBD are similar to those of other countries. UC is more common than EC. There is a higher proportion of women with the disease. The severity of the disease was higher among HCUCH (Rev Méd Chile 2005; 133: 1295-304).

(Key Words: Colitis, ulcerative, Crohn disease; Inflammatory bowel disease)

Recibido el 11 de marzo, 2005. Aceptado el 13 de junio, 2005.

${ }^{1}$ Servicio de Medicina Interna, Hospital Clínico de la Universidad de Chile, ${ }^{2}$ Servicio de Gastroenterología, Clínica Las Condes, ${ }^{3}$ Sección de Gastroenterología, ${ }^{4}$ Servicio de Cirugía, Hospital Clínico de la Universidad de Chile, Santiago de Chile.

$E^{1}$ término "Enfermedades Inflamatorias Intestinales" (EII) incluye las entidades colitis ulcerosa (CU), enfermedad de Crohn (EC) y colitis indeterminada (CI). Si bien son entidades poco frecuentes, contribuyen en forma importante a la patología colorrectal por su cronicidad, severidad, evolución con recaídas, afecta la calidad de vida, alta morbilidad quirúrgica, limitada eficacia terapéutica médica y por tener mayor riesgo de desarrollar cáncer colorrectal ${ }^{1,2}$. Aunque la etiolo-

Correspondencia a: Dra. Carolina Figueroa C. Bustos 2730, Depto. 304, Providencia. Fax: 4612713.

E-mail: cfigueroacorona@yahoo.com gía de las EII permanece aún desconocida, se reconoce que factores genéticos, inmunológicos y ambientales tienen un papel importante en la patogénesis de esta enfermedad ${ }^{3-7}$. La mayor prevalencia se encuentra actualmente en Europa y Estados Unidos de Norteamérica (EE.UU.) donde la incidencia para CU en Norteamérica es entre 2,2 y 14,3 casos por 100.000 y de 3,1 a 14,6 casos por 100.000 de $\mathrm{EC}^{3,4}$. En los países europeos la incidencia alcanza 1,5 a 20,3 casos por 100.000 para CU y 0,7 a 9,8 casos por 100.000 para $E^{8-14}$. Es difícil estimar la incidencia y prevalencia de las EII en Chile por la falta de estandarización de los criterios diagnósticos, lo que se traduce en subdiag- 
nóstico y falla en la derivación a centros con mayor experiencia en el diagnóstico y manejo ${ }^{15-16}$. Otro aspecto importante es el aumento en la incidencia de EII en todo el mundo $3,4,13,17-22$, sobre todo en los países en vías de desarrollo. Un aspecto interesante y no claramente explicado es que en la medida que los países han mejorado sus condiciones socioeconómicas los casos de EII también han aumentado 11,17,23-25. Esta situación se presenta en Europa y en América Latina ${ }^{1,25-27}$ y existe la impresión, no bien documentada, de que esta situación ocurre también en Chile. Esto ha llevado a pensar que existe una estrecha relación entre EII y factores ambientales como son la contaminación, procesamiento de alimentos, industrialización, vacunación y cambios dietéticos ${ }^{3,28}$. Este aumento en la incidencia ha determinado también un incremento en el costo económico global debido al alto precio del tratamiento, que habitualmente es prolongado, y manejo de las complicaciones de las EII. Más aún, a menudo se requiere de una intervención multidisciplinaria, lo que eleva aún más los $\operatorname{costos}^{29}$. De hecho, esta consecuencia económica de las EII ha motivado en algunos países un mayor interés en la patogenia y en la búsqueda de nuevas medidas terapéuticas.

Uno de los objetivos de este trabajo fue describir las manifestaciones clínicas de un número considerable de pacientes chilenos con EII, para caracterizar sus formas de presentación, evaluando las manifestaciones extraintestinales, evolución, complicaciones y resultados de las terapias. Otro objetivo fue evaluar la influencia que pueden tener los factores socioeconómicos en su tratamiento y curso clínico, dado que los pacientes fueron controlados en dos centros médicos que atienden a pacientes con situaciones socioeconómicas distintas (Clínica Las Condes y Hospital Clínico de la Universidad de Chile).

\section{PACIENTES Y MÉTODOS}

Se realizó un estudio descriptivo y retrospectivo de 282 pacientes mayores de 13 años, diagnosticados como EII, atendidos en el Hospital Clínico de la Universidad de Chile (HCUCH) y en la Clínica Las Condes (CLC) entre enero de 1990 y agosto de 2002. Se analizaron los síntomas de presentación, el tiempo entre aparición de los síntomas y el diagnós- tico, principales exámenes diagnósticos tales como endoscopias, imagenología, biopsias, los tratamientos indicados, la evolución de la enfermedad, la presencia de manifestaciones extraintestinales, complicaciones y manejo de ellas. Se diagnosticó CU en aquellos pacientes que, además de presentar un cuadro clínico sugerente, contaban con alteraciones endoscópicas características del colon, que en todos los casos incluía el recto con hiperemia y edema de la mucosa, la que se observó granular y friable en forma continua y la presencia de ulceraciones o pseudopólipos y en quienes se habían descartado causas inflamatorias específicas o infecciosas bacterianas o parasitarias. Los pacientes contaban con biopsias compatibles con el diagnóstico ${ }^{30}$. Estos pacientes se clasificaron según Truelove-Witts ${ }^{31}$ en leves, moderados y severos. Se consideraron portadores de EC a aquellos pacientes que presentaban un cuadro clínico sugerente y que tenían alteraciones del intestino delgado, colon o ambos, con presencia de úlceras aftoideas, estenosis, fístulas, fisuras, compromiso perianal, lesiones que frecuentemente altemaban con mucosa aparentemente sana, asociado a una biopsia altamente sugerente ${ }^{32}$. La localización y extensión del compromiso de CU o EC se determinó por la endoscopia, exámenes por imágenes o ambos. Para el análisis estadístico de los datos se utilizó el test t de Student y $\mathrm{X}^{2}$.

\section{RESULTADOS}

Doscientos ochenta y dos casos satisfacían los criterios diagnósticos. Cuarenta y cuatro fueron excluidos por no tener datos suficientes. Ciento ochenta y un pacientes (76\%) fueron diagnosticados como CU ( $\bar{x}$ edad: 30 años, rango 14-78 años), de ellos 104 (57\%) eran mujeres. Los restantes 57, portaban EC ( $\bar{x}$ edad: 39 años, rango $11-57$ años); 39 (76\%) de ellos eran mujeres. La relación CU/EC fue 3,2:1. Aproximadamente la mitad de los pacientes (48\%) tenían entre 20 y 39 años de edad en el momento del diagnóstico. Cien pacientes con CU fueron atendidos en HCUCH y 81, en CLC, en tanto que 20 pacientes con $\mathrm{EC}$ fueron atendidos en HCUCH y 37 en CLC. Con respecto a la fecha de diagnóstico, en el caso de los pacientes con EC, 69\% de ellos fue diagnosticado después del año 1995, es decir en la segunda mitad del período estudiado. El $59 \%$ de los pacientes con 
CU fueron diagnosticados después de esa fecha (Figura 1).

Pacientes con colitis ulcerosa. Al momento del diagnóstico, los síntomas sugerentes de CU habían estado presentes por una media de 13 meses (rango 1-120 meses), sin diferencias significativas entre los dos centros. El síntoma principal al inicio fue la rectorragia o disentería $(35 \%)$, diarrea $(27 \%)$, dolor abdominal (14\%), pujo, tenesmo o ambos (12\%), fiebre, compromiso del estado general o ambos (5\%), baja de peso (4\%), náuseas o vómitos (1\%) y otras manifestaciones misceláneas (2\%). La extensión de la colitis fue: proctitis, $21 \%$; rectosigmoiditis, $30 \%$; colitis izquienda, $21 \%$; y solamente $28 \%$, presentaba pancolitis con compromiso proximal al ángulo esplénico (Tabla 1). La severidad de la colitis

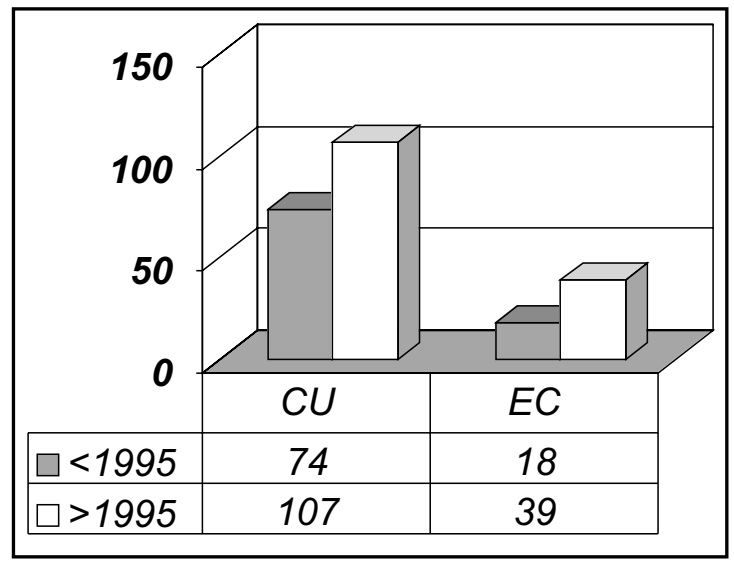

FIGURA 1. Número de pacientes diagnosticados antes y después de 1995.

Tabla 1. C aracterísticas de los pacientes portadores de colitis ulcerosa (CU)

\begin{tabular}{|c|c|c|c|c|}
\hline & & HCUCH & CLC & Total \\
\hline CU & & n: $100(\%)$ & n: $81(\%)$ & n: 181 (\%) \\
\hline Localización & & & & \\
\hline Proctiti & & $10(10)$ & $28(34)$ & $38(21)$ \\
\hline Rectosi & moiditis & $41(41)$ & $14(17 \%)$ & $55(30)$ \\
\hline Colitis & zquierda & $11(11)$ & $27(33)$ & $38(21)$ \\
\hline Pancoli & & $38(38)^{*}$ & $12(15)$ & $50(28)$ \\
\hline Complicacio & & 7 (7) & $6(7)$ & $13(7)$ \\
\hline Hemor & agia digestiva & $2(28)$ & $2(33)$ & $4(31)$ \\
\hline Megacc & lon & 0 & 1 (17) & $1(8)$ \\
\hline Colecci & fn o absceso & $3(43)$ & $1(17)$ & $4(31)$ \\
\hline Estenos & & $1(14)$ & 0 & $1(8)$ \\
\hline Perfora & ión & $1(14)$ & 0 & $1(8)$ \\
\hline Pouchi & & 0 & $2(33)$ & $2(15)$ \\
\hline Requirieron & irugía & $26(26)^{*}$ & $7(9)$ & $33(18)$ \\
\hline Manifestacio & es extraintestinales & $10(10)$ & $23(28)$ & 33 (18) \\
\hline Órgano & Alteración & & & \\
\hline Piel & $\begin{array}{l}\text { Eritema } \\
\text { nodoso }\end{array}$ & $4(4)$ & $4(5)$ & $8(4)$ \\
\hline & P gangrenoso & & & \\
\hline Articular & Artritis & $10(10)$ & $17(21)$ & $27(15)$ \\
\hline & Artralgia & & & \\
\hline Mucosa oral & Úlceras & $2(2)$ & $2(2)$ & $4(2)$ \\
\hline Oculares & Epiescleritis & $3(3)$ & $1(1)$ & $4(2)$ \\
\hline & Ojo seco & & & \\
\hline
\end{tabular}

${ }_{\mathrm{p}}^{*}<0,01$ cuando se compararon pacientes atendidos HCUCH y CLC.

HCUCH: Hospital Clínico de la Universidad de Chile. CLC: Clínica Las Condes. 
fue similar en los dos centros. Veintinueve porciento de los pacientes controlados en el HCUCH fueron catalogados de severos y $23 \%$ lo fuemon en CLC. En contraste, la extensión de la colitis fue mayor en los pacientes del HCUCH ya que $38 \%$ presentaba pancolitis, mientras sólo la presentaban $15 \%$ de los pacientes de CLC ( $p \varangle 0,008$ ) (Tabla 1). Los pacientes con pancolitis del HCUCH requirieron con más frecuencia cirugía que los pacientes de CLC y presentaron mayor tiempo de latencia entre la aparición de los síntomas y el diagnóstico (14,9 meses v/s 4 meses en CLC). Las complicaciones de la CU fueron similares entre los centros (Tabla 1).

En 33 pacientes (18\%) con CU fue necesario realizar algún procedimiento quirúrgico, registrándose un fallecimiento como consecuencia de complica- ciones postquirúrgicas. La cirugía fue más frecuente en los pacientes del HCUCH al compararlos con los de CLC ( $p<0,005$ IC: $95 \%$ ), lo que sugiere mayor severidad de la enfermedad (Tabla 1). En cuanto al tratamiento que recibieron los pacientes portadores de CU durante las crisis, hubo un uso significativamente mayor de 5-ASA en los pacientes atendidos en CLC en comparación a los de HCUCH ( $p$ $\varangle 0,0001)$ y hubo una tendencia a usar más frecuentemente esteroides en HCUCH comparado con los pacientes de CLC, aunque esta última diferencia no fue significativa (Figura 2). En el tratamiento de mantención de la $\mathrm{CU}$, los pacientes de CLC recibieron más frecuentemente derivados de la 5-ASA (5ASA) ( $p<0,0001$ ) que los pacientes de HCUCH y significativamente menos esteroides que los pacien-

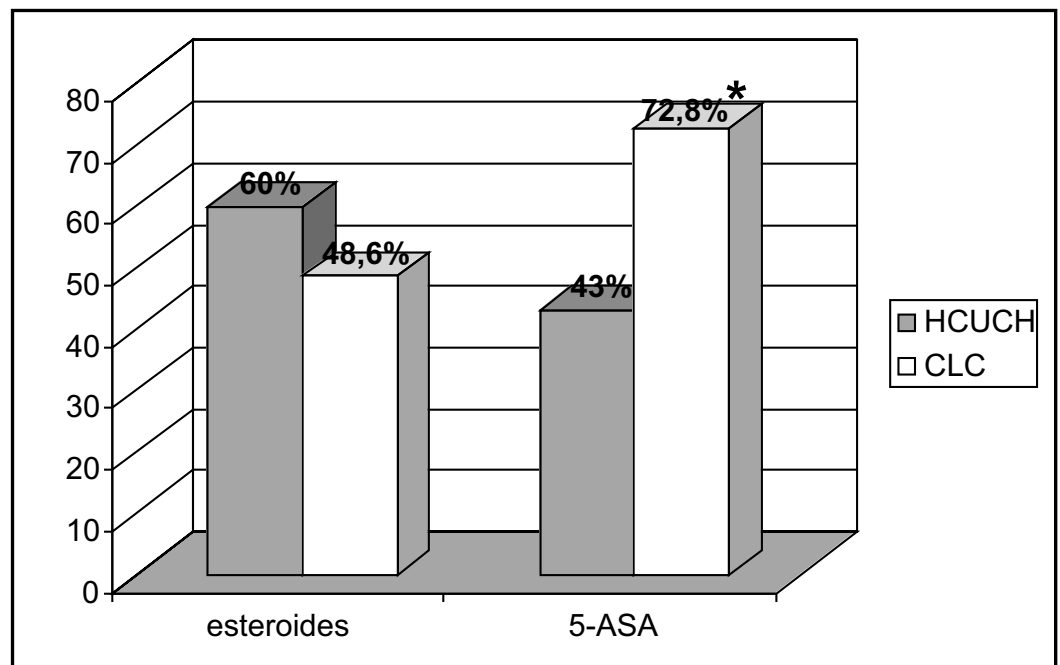

FiguRA 2. Tratamiento de crisis de colitis ulcerosa según centro.

Figura 3. Tratamiento de mantención de colitis ulcerosa según centro.

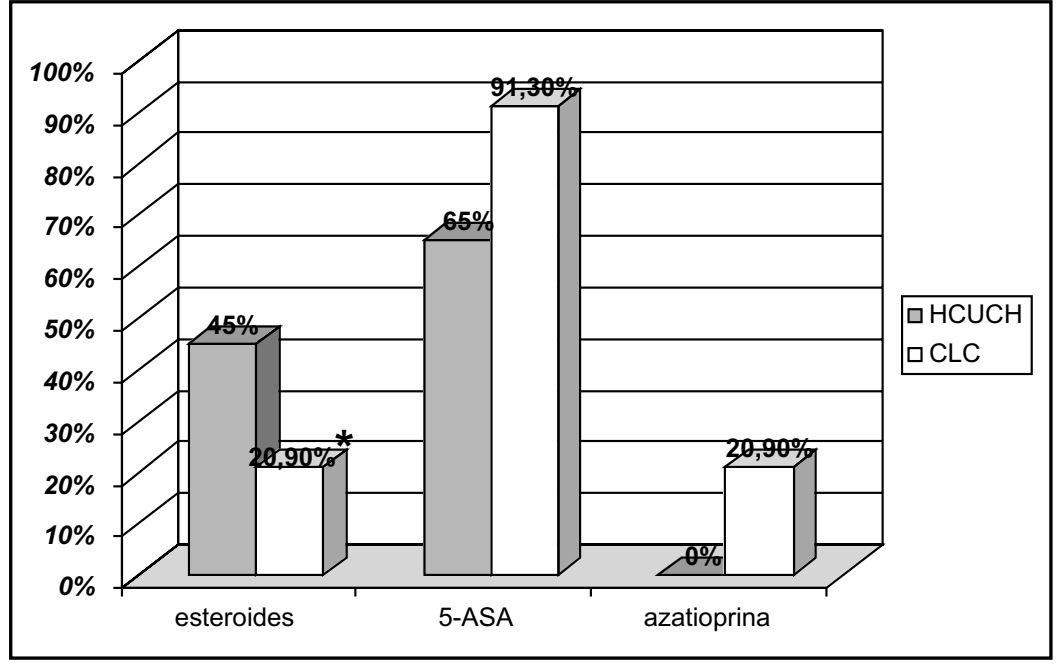


tes de HCUCH $(p<0,001)$. Con respecto al uso de azatioprina (AZA), cabe destacar que ningún paciente con CU de HCUCH recibió esta terapia como mantención, en cambio 20\% de los tratados en CLC recibieron AZA (Figura 3).

Pacientes con enfermedad de Crohn. El período que medió entre la aparición de síntomas y el diagnóstico de EC fue, en promedio, de 17 meses (1-136 meses), siendo levemente mayor en pacientes atendidos en $\mathrm{HCUCH}$, pero sin diferencia significativa al compararlo con el tiempo que medió en los pacientes atendidos en CLC.

Las manifestaciones iniciales en los pacientes con EC fueron, principalmente, dolor abdominal (73\%), diamea (49\%) y hematoquezia o disentería (39\%).
La extensión del compromiso del tubo digestivo en EC se muestra en la Tabla 2, destacando 2 casos (4\%) con compromiso esofágico comprobado. Los pacientes con EC tratados en $\mathrm{HCUCH}$ presentaron más frecuentemente complicaciones (85\%), a diferencia de los pacientes atendidos en CLC (30\%), lo que representa una diferencia estadísticamente significativa $(\mathrm{p}<0,0001)$ (Tabla 2). En total 16/57 pacientes (57\%) con EC requirieron cirugía para manejo de las complicaciones y uno falleció por complicaciones postquirúrgicas. Si bien un mayor número de pacientes con EC tratados en el HCUCH necesitó cirugía para su manejo, no hubo diferencias en este aspecto entre los centros en cuanto a la necesidad de cirugía en EC.

Tabla 2. C aracterísticas de los pacientes portadores de enfermedad de C rohn (EC)

\begin{tabular}{|c|c|c|c|c|}
\hline & & $\mathrm{HCUCH}$ & CLC & Total \\
\hline $\mathrm{EC}$ & & n: 20 & $\mathrm{n}: 37$ & $\mathrm{n}: 57$ \\
\hline Localización & & & & \\
\hline Ileon & & $3(15)$ & $18(49)$ & 21 (37) \\
\hline Colon & & $12(60)$ & $15(40)$ & 27 (47) \\
\hline Ileocols & & $5(25)$ & $2(5)$ & 7 (19) \\
\hline Esófagc & & 0 & $2(5)$ & $2(4)$ \\
\hline Complicacios & & $17(85)^{*}$ & $11(30)$ & $28(76)$ \\
\hline Colección o & absceso & $2(12)$ & 0 & $2(7)$ \\
\hline Fístula & & $6(35)$ & $4(36)$ & $10(36)$ \\
\hline Obstruc & ción & $1(6)$ & $6(54)$ & $7(25)$ \\
\hline Perfora & iión & $2(12)$ & $1(9)$ & $3(11)$ \\
\hline Estenos & & $5(29)$ & 0 & $5(18)$ \\
\hline Pouchit & & $1(6)$ & 0 & $1(3)$ \\
\hline Requirieron & irugía & $9(45)$ & $7(19)$ & $16(57)$ \\
\hline Manifestacio & es extraintestinales & $4(20)$ & $11(30)$ & $15(37)$ \\
\hline Órgano & alteración & & & \\
\hline Piel & Eritema nodoso & $2(10)$ & $3(8)$ & $5(9)$ \\
\hline & P gangrenoso & & & \\
\hline Articular & Artritis & $1(5)$ & $7(19)$ & $8(14)$ \\
\hline & Artralgia & & & \\
\hline Mucosa oral & Úlceras & $2(10)$ & $2(5)$ & $4(7)$ \\
\hline Oculares & Epiescleritis & 0 & $1(3)$ & $1(2)$ \\
\hline & Ojo seco & & & \\
\hline
\end{tabular}

${ }^{*} \mathrm{p}<0,01$ cuando se compararon pacientes atendidos en HCUCH y CLC.

HCUCH: Hospital Clínico de la Universidad de Chile. CLC: Clínica Las Condes. 
$\mathrm{Al}$ analizar el tratamiento de EC, también hay una tendencia a mayor uso de 5-ASA en los pacientes atendidos en CLC, durante las crisis (81\%) y como terapia de mantención $(94,5 \%)$. En HCUCH se usó 5ASA en $11,1 \%$ de las crisis y $70 \%$ mantención. Al comparar el uso de 5-ASA en los dos centros, este resultó significativo sólo durante el tratamiento de las crisis ( $p<0,0001$ ). Con respecto a los esteroides, éstos fueron usados en período de crisis en mayor proporción en CLC (68\% vs $55 \%$ de $\mathrm{HCUCH}$ ), no así en terapia de mantención, donde nuevamente se utilizan más en $\mathrm{HCUCH}$ ( $50 \%$ vs 30\%) (Figuras 4 y 5). Estas diferencias no fueron significativas. Tampoco hubo diferencias con el uso de AZA como terapia de mantención de EC entre ambos centros. Hubo dos casos de EC que recibieron terapia de mantención con infliximab, uno en cada centro.

Se diagnosticaron manifestaciones extraintestinales en 48/238 (20\%) pacientes estudiados. Los más frecuentes fueron compromiso articular, seguido de compromiso de piel, de mucosa oral y ocular. La distribución de estas manifestaciones por patología y por centro se muestran en las Tablas 1 y 2. En los portadores de CU las manifestaciones articulares fueron significativamente mayor que las otras ( $p<0,0001$ ). En los pacientes con EC no hubo diferencias en cuanto al área comprometida. En los pacientes con EC no hubo diferencia entre las manifestaciones extraintestinales, esto probable-

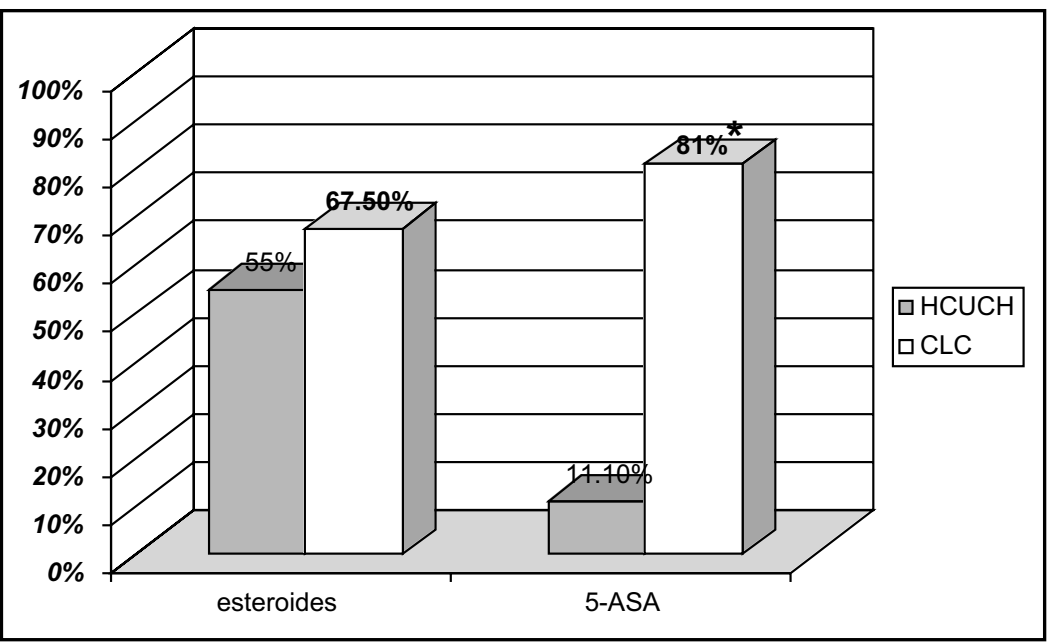

FIGURA 4. Tratamiento de crisis de enfermedad de Crohn según centro.

FIGURA 5. Tratamiento de mantención de enfermedad de Crohn según centro.

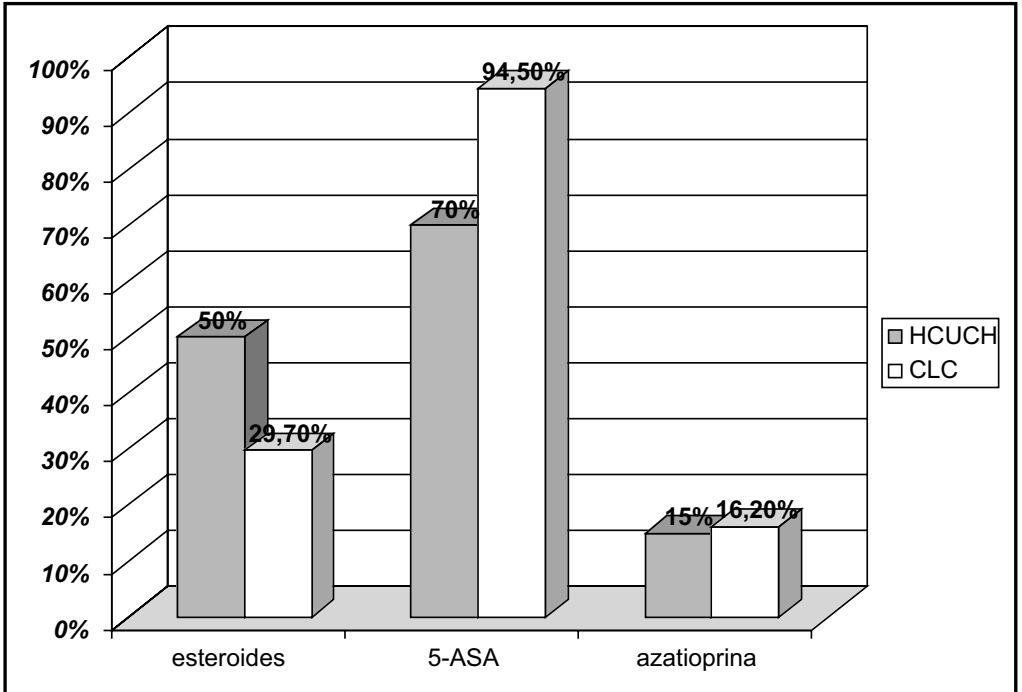


mente se debe al número reducido de pacientes. Aunque las manifestaciones extraintestinales fueron más frecuentes en los pacientes con EC (37\% vs $18 \%)$, esta diferencia no fue significativa.

\section{DisCUSIÓN}

Basado en los resultados de este estudio, las características de EII en Chile son similares a las descritas en otros países. Las EII son más frecuentes en el sexo femenino y se presentan principalmente en el adulto joven ${ }^{1,17-19,33-38}$. El hecho que la mayoría de los casos fueran diagnosticados después del año 1995 podría sugerir una tendencia al aumento del diagnóstico de estas enfermedades en los últimos años, lo que también ocurre en otros países ${ }^{1,8,17}$. Esto podría explicarse por un aumento real en la incidencia o por una mayor precocidad en el diagnóstico, ya sea porque la población consulta más precozmente, o hay un mayor conocimiento médico de la enfermedad y por lo tanto un mejor y más temprano diagnóstico de ella. Este aspecto, sin embargo, debe mejorar, dada la latencia media entre 13 y 17 meses que encontramos en nuestros pacientes. Es muy probable que, como en otros países, exista un aumento real de la incidencia de EII, la que debe documentarse haciendo estos diagnósticos comunicables a las autoridades de salud. Este aumento podría estar relacionado con la exposición a los factores ambientales propios de un país en vías de desarrollo como el nuestro ${ }^{17}$. Es de interés que en nuestra población la CU fue 3 veces más frecuente que EC, relación que se observaba anteriormente en países desarrollados y que últimamente ha ido cambiando por mayor frecuencia de EC.

La presencia de manifestaciones extraintestinales fue, en esta serie, menor que lo descrito en casuísticas extranjeras ${ }^{26,35,39}$, pero cabe considerar que por tratarse de un estudio retrospectivo, puede existir un sesgo importante y no haberse enfatizado el compromiso articular o de otras manifestaciones. De acuerdo con otras series las manifestaciones extraintestinales ocurren más frecuentemente en $\mathrm{EC}$ que en $\mathrm{CU}^{33}$.

En los pacientes con CU el compromiso más frecuente descrito fue pancolitis (28\%) lo que sugiere mayor severidad y cronicidad ${ }^{17,33,40}$. Llama la atención también que $18 \%$ de nuestros pacientes con CU requirieron cirugía, similar a lo descrito en series extranjeras ${ }^{26}$. Esto sugiere desconocimiento de los beneficios de una terapia médica de mantención apropiada, poca accesibilidad de los pacientes a los medicamentos indicados o mal seguimiento de estos casos. Tanto los médicos tratantes como los pacientes deben ser educados sobre el carácter crónico y recurrente de las EII y de los beneficios del tratamiento de mantención en la prevención de las crisis y de las complicaciones.

En los pacientes portadores de EC la localización también presentó diferencias con respecto a lo descrito en la literatura, donde se señala que es principalmente ileocolónica $(40-50 \%)^{8,17,26,33,41}$, en cambio, en la presente serie la localización más frecuente fue colónica. Probablemente, podría deberse a la falta de estudios endoscópicos o radiológicos del íleon y que no se hayan detectado lesiones a este nivel. Es importante plantear en este punto que el acceso de un mayor número de pacientes a la colonoscopia con ileoscopia, estudio de imágenes y eventualmente el uso de la cápsula endoscópica pueden ayudar a precisar el compromiso ileal ${ }^{42-43}$. Destacamos la presencia de $4 \%$ de compromiso esofágico, cuando en la literatura se describe esta localización en menos de $1 \%$. Con respecto a las complicaciones, las series internacionales describen $35 \%$ de ellas ${ }^{17}$, mientras en nuestra población ello ocurrió en $75,6 \%$, considerablemente más alto. Esta diferencia se hace más evidente al comparar ambos centros donde los pacientes atendidos en $\mathrm{HCUCH}$ tuvieron significativamente más complicaciones, lo que sugiere un manejo menos adecuado. De los pacientes que presentaron complicaciones $57 \%$ requirió tratamiento quirúrgico, similar a lo descrito en otras series que alcanza $60 \%{ }^{8}$.

Es importante señalar que existen importantes diferencias socioeconómicas entre los centros hospitalarios estudiados. El HCUCH atiende una población de recursos económicos medios, no así en cuanto a recursos técnicos y humanos. En cambio, los que consultan en CLC pertenecen al estrato socioeconómico alto, lo que sugiere mayor nivel de educación y disponibilidad de recursos terapéuticos. Estas diferencias se reflejan en que los pacientes controlados en $\mathrm{HCUCH}$ presentan patologías más severas, mayor número de complicaciones y requieren más cirugía para su manejo. 
En el caso de los pacientes con pancolitis del HCUCH fueron los que más requirieron de cirugía para su manejo, en cambio los pacientes con pancolitis de CLC no requirieron más cirugías que los que tenían colitis de menor extensión. Además, este grupo del HCUCH tuvo un tiempo de latencia de diagnóstico casi 10 meses mayor que los pacientes con pancolitis de CLC. Esto sugiere que los pacientes de $\mathrm{HCUCH}$ presentaron una evolución más severa porque se diagnosticaron más tardíamente. También se observó que los pacientes del HCUCH recibían menos 5-ASA que los pacientes de CLC y, a diferencia de ellos, recibían mucho más terapia esteroidal tanto durante las crisis, como en terapia de mantención. Esto puede deberse a razones económicas, ya que los esteroides orales (prednisona) son de bajo costo y de mayor disponibilidad. Sin embargo su efectividad en la mantención de las remisiones es cuestionable $^{44}$. Además los pacientes estudiados en HCUCH no sólo usaron menos 5-ASA, sino que las dosis utilizadas fueron menores que las usadas por los pacientes de CLC (datos no mostrados), lo que sugiere menor eficacia al mantener la EII en remisión y prevención de las complicaciones. Esto también puede deberse a causas económicas, lo que a impresión de los autores determina una diferencia muy importante en la adherencia al tratamiento siendo mucho menor en $\mathrm{HCUCH}$. Otra diferencia entre los dos centros, es el hecho de que ningún paciente con $\mathrm{CU}$ del $\mathrm{HCUCH}$ recibió azatioprina (AZA) como terapia de mantención, mientras en CLC, 10\% la recibía. Esta diferencia terapéutica no puede invocarse como

\section{REFERENCIAS}

1. Russel MG, Stockbrugger RW. Epidemiology of Inflammatory Bowel Disease: an update. Scand J Gastroenterol 1996; 31: 417-27.

2. SHAH SA, PEPPERCORN MA. Inflammatory bowel disease therapy: an update. Compr Ther 1995; 21: 296-302.

3. Loftus EV JR, SANDBorn WJ. Epidemiology of Inflammatory bowel disease. Gastroenterol Clin North Am 2002; 31: 1-20.

4. LofTus EV JR. Clinical epidemiology of inflammatory bowel disease: Incidence, prevalence, and environmental influences. Gastroenterology 2004; 126: 1504-17. causa de la peor evolución de los pacientes del HCUCH ya que el beneficio de la AZA en el tratamiento de la EC está bien establecido, mientras que en CU sigue siendo controversial ${ }^{45}$.

Estas diferencias en el curso de las EII entre los dos centros médicos enfatiza la necesidad de mejorar la información de los médicos por una parte y la disponibilidad de exámenes adecuados y medicamentos apropiados. En este punto, pensamos que la recomendación terapéutica debe ser el uso de derivados de la 5-ASA en dosis óptimas y el uso de AZA o 6-mercaptopurina en los casos más resistentes y evitar el uso de esteroides para mantener la enfermedad en remisión, por no haber beneficio demostrado en su uso y por los efectos colaterales que estos producen ${ }^{44}$.

Aunque no es posible concluir en base a este estudio que la incidencia de EII haya aumentado en Chile, podemos suponer que esto está ocurriendo, basados en la experiencia de los autores, lo que además concuerda con observaciones en todo el mundo. Este hecho, sumado a los altos costos sociales y económicos de esta patología debiera implicar un aumento en el conocimiento de las EII basado en estudios prospectivos y multicéntricos que nos permita delinear más a fondo las características de las enfermedad inflamatorias intestinales en nuestra región. Esto permitiría optimizar el diagnóstico precoz y una terapia adecuada, mejorando el pronóstico de estos pacientes. Debe ser motivo de preocupación de parte de la comunidad médica el acceso de parte de los pacientes a métodos diagnósticos adecuados y las terapias necesarias.

5. Podolsky DK. Inflammatory bowel disease (1). N Engl J Med 1991; 325: 928-37.

6. Blumberg S, Warnen S. Prospects for research in inflammatory bowel disease. JAMA 2001; 285: $643-47$

7. Monsen U, Bernell O, Johansson C, Helers G. Prevalence of inflammatory bowel disease among relatives of patients with Crohn's disease. Scand J Gastroenterol 1991; 26: 302-6.

8. Lakatos L, Mester G, Erdely Z, Balogh M, Szipocs I, KAMARAS $G$ et AL. Striking elevation in incidence and prevalence of inflammatory bowel disease in a province of western Hungary between 19772001. World J Gastroenterol 2004; 10: 404-9. 
9. Fonager $\mathrm{K}$, Sorensen Ht, Olsen J. Change in Incidence of Crohn's disease and ulcerative colitis in Denmark. A study based on the national registry of patients, 1981-1992. Int J Epidemiol 1997; 26: 1003-8.

10. Shivananda S, Pena AS, Mayberry JF, Ruitenberg EJ, HOEDEMAEKER PJ. Epidemiology of proctocolitis in the region of Leiden, The Netherlands. A population study from 1979-1983. Scand J Gastroenterol 1987; 22: 993-1002.

11. Helers G. Crohn's disease in Stockholm county, 1955-1974: a study of epidemiology, result of surgical treatment and long term prognosis. Acta Chir Scand Suppl 1979; 490: 1-84.

12. Berner J, KiAer T. Ulcerative colitis and Crohn's disease on the Faroe Islands 1964-1983. A retrospective epidemiological survey. Scand J Gastroenterol 1986; 21: 188-92.

13. Stonnington CM, PhiшPS SF, Melton LJ $3^{\text {RD }}$, ZinsmeisTER AR. Chronic ulcerative colitis: Incidence and prevalence in a community. Gut 1987; 28: 402-9.

14. Shivananda S, Lennard-Jones J, Logan R, Fear N, Price A, Carpenter L et al. Incidence of inflammatory bowel disease across Europe: is there a difference between north and south? Results of the European Collaborative Study on Inflammatory Bowel Disease (EC-IBD). Gut 1996; 39: 6907.

15. Ilabaca M, Toledo C, Jiménez P. Colitis ulcerosa idiopática: Una enfermedad mejor reconocida. Gastr Latinoam 2001; 12: 274 (resumen).

16. IbáÑEZ P, Vergara MT. Enfermedad inflamatoria intestinal: Características clínicas de una población en control. Gastr Latinoam 2001; 12: 275 (resumen).

17. Rodrigo L, Riestra S, Niño P, Cadahía V, Tojo R, FuENTES D ET AL. A population-based study on the incidence of inflammatory bowel disease in Oviedo (Northern Spain). Rev Esp Enferm Dig 2004; 96: 296-305.

18. Rose JD, Roberts GM, Wiшums G, MayberRy JF, RHodes J. Cardiff Crohn's disease jubilee: the incidence over 50 years. Gut 1988; 29: 346-51.

19. Sediack RE, Whisnant J, Elveback LR, Kurland LT. Incidence of Crohn's disease in Olmsted County, Minnesota, 1935-1975. Am J Epidemiol 1980; 112: 759-63.

20. SONNENBERG A. Geographic variation in the incidence of and mortality from inflammatory bowel disease. Dis Colon Rectum 1986; 29: 854-61.
21. Munkholm P, Langholz E, Nielsen OH, Kreiner S, BINDER V. Incidence and prevalence of Crohn's disease in the county of Copenhagen, 1962-87: a sixfold increase in incidence. Scand J Gastroenterol 1992; 27: 609-14.

22. Leong RW, Lau JY, Sung JJ. The epidemiology and phenotype of Crohn's disease in the Chinese population. Inflamm Bowel Dis 2004; 10: 646-51.

23. NuNES GC, AHLQUIST RE JR. Increasing incidence of Crohn's disease. Am J Surg 1983; 145: 578-81.

24. Thomas GA, Miliar-Jones D, Rhodes J, Roberts GM, Wiwams GT ET aL. Incidence of Crohn's disease in Cardiff over 60 years: 1986-1990 an update. Eur J Gastroenterol Hepatol 1995; 7: 401-5.

25. Appleyard C, Hernández G, Ríos-Bedoya CF. Basic epidemiology of inflammatory bowel disease in Puerto Rico. Inflamm Bowel Dis 2004; 10; 106-11.

26. Souza MH, Troncon LE, Rodrigues CM, Viana CF, Onofre PH, Monteiro RA ET aL. Trends in the occurrence (1980-1999) and clinical features of Crohn's disease and ulcerative colitis in a university hospital in Southeastern Brazil. Arq Gastroenterol 2002; 39: 98-105.

27. unares de la Cal JA, Canton C, Hermida C, PérezMiranda M, Mate-JimÉnEZ J. Estimated incidence of inflammatory bowel disease in Argentina and Panama (1987-1993). Rev Esp Enferm Dig 1999; 91: 277-86.

28. Мочм B, Еквом A. Epidemiology of inflammatory bowel disease-methodological considerations. Dig Liver Dis 2002; 34: 364-9.

29. HAY JW, HaY AR. Inflammatory bowel disease: cost-of-illness. J Clin Gastroenterol 1992; 14: 30917.

30. JewEL DP. Ulcerative Colitis In; Feldman M, Scharschmidt BF, Sleisenger MH, editors. Sleisenger \& Fordtron's Gastrointestinal and liver disease. Patophysiology, diagnosis and managment. $6^{a}$ ed Philadelphia: WB Saunders; 1998; 1735-61.

31. Seo M, Okada M, Yao T, Okabe N, Maeda K, Оh K. Evaluation of disease activity in patients with moderately active colitis: comparisons between a new activity index and Truelove and Witt's classification. Am J Gastroenterol 1995; 90: 1759-63.

32. Kronluth, Sachar DK, Salomon P. Crohn's disease. In: Feldman M, Scharschmidt BF, Sleisenger MH, editors. Sleisenger \& Fordtron's Gastrointestinal and Liver Disease. Patophysiology, diagnosis and managment. $6^{\underline{a}}$ ed Philadelphia: WB Saunders; 1998; 1735-61. 
33. Lakatos L, Pandur T, David G, Balogh Z, Kuronya $P$, TOLAS A ET AL. Association of extraintestinal manifestations of inflammatory bowel disease in a province of Western Hungary with disease phenotype: Results of a 25-year follow-up study. World J Gastroenterol 2003; 9: 2300-7.

34. TRiantafimdis JK, Emmanouildis A, Manousos ON, Pomonis E, Tsitsa C, Cheracakis P ET al. Ulcerative colitis in Greece: clinicoepidemiological data, course and prognostic factors in 413 consecutive patients. J Clin Gastroenterol 1998; 27: 204-10.

35. Kronbluth A, Sachar DK, Salomón P. Crohns disease. In: Feldman M, Scharschmidt BF, Sleisenger MH, editors. Sleisenger \& Fordtron's Gastrointestinal and liver disease. Patophysiology, diagnosis and managment. 6a ed Philadelphia: WB Saunders; 1998; 1708-34.

36. Riegier G, Tartaglione MT, Carratú R, D’Inca R, Valpiani D, Russo MI et al. Age -related clinical severity at diagnosis in 1705 patients with ulcerative colitis. A study by GISC (Italian ColonRectum Study Group). Dig Dis Sci 2000; 45: 462-5.

37. Gower-Rousseau C, Salomez JL, Dupas JL, Marti R, NutTens MC, VotTe A Et al. Incidence of inflammatory bowel disease in Northern France (19881990). Gut 1994; 35: 1433-8.

38. TRIANTAFILdis JK, EMmanouildis A, Manousos O, Nicolakis D, Kogevinas M. Clinical patterns of Crohn's disease in Greece: a follow-up study of 155 cases. Digestion 2000; 61: 121-8.

39. Mintz R, Feluer ER, Bahr RL, Shah SA. Ocular manifestations of inflammatory bowel disease. Inflamm Bowel Dis 2004; 10: 135-9.
40. Herfarth $H$, Rogler G. Inflammatory bowel disease. Endoscopy 2005; 37: 42-7.

41. Jensen C, Valuejo J, Vergara Ji, Azolas C, Pérez G, GaRRIDo R ET AL. Enfermedad de Crohn y cirugía. Rev Chil Cir 1997; 49: 82-7.

42. Daperno M, Sostegni R, Lavagna A, Crocella L, Ercole E, Rigazio C et al. The role of endoscopy in inflammatory bowel disease. Eur Rev Med Pharmacol Sci 2004; 8: 209-14.

43. LennaRd-Jones JE, Shivananda S. Clinical uniformity of inflammatory bowel disease a presentation and during the first year of disease in the north and south of Europe. Eur J Gastroenterol Hepatol 1997; 9: 353-9.

44. Valera JM, Poniachik J, Valenzueia J. Enfermedad de Crohn: Enfoque terapéutico. Gastr Latinoam 2002; 4: 270-80.

45. DuBINSKY MC. Azathioprine, 6-mercaptopurine in inflammatory bowel disease: pharmacology, efficacy and safety. Clin Gastroenterol Hepatol 2004; 2: 731-3.

46. Hildsen R, Verhoef M, Best A, Pocobe山 G. A national survey on the patterns of treatment of inflammatory bowel disease in Canada. BMC Gastroenterology 2003; 3: 10.

Agradecimientos

Agradecemos la colaboración del Dr. Renato Palma en la revisión de este trabajo. 